\title{
Multi-Parametric Analysis of Intrinsic Cell Death Using Live Cell Microscopy
}

\author{
Gaurav N. Joshi and David A. Knecht \\ Department of Molecular and Cell Biology, University of Connecticut, Storrs, USA
}

Microscopy has come a long way with complete automation and faster acquisition rates. Simultaneously, development of a variety of genetically encoded fluorescent probes or organic probes to detect specific cellular processes and changes in physiological parameters during cell death processes have also advanced. Using a combination of these tools, we report a semi- high throughput method to detect cell death in alveolar macrophages stimulated with compounds (silica particles, staurosporine and Leu-Leu-O-Me) that activate the intrinsic cell death pathway. We have found that for every treatment, cells followed a similar pathway to death, however, each cell proceeds towards death in a temporally distinct manner.

Cell death is necessary to maintain homeostasis; however, under several conditions this homeostasis is disturbed resulting in adverse physiological and pathological outcomes. Traditionally cell death has been characterized in to be either apoptosis or necrosis. Valuable research in the field of cell death has further led to the characterization of more narrowly defined pathways by which cells die, and these are linked to various diseases [1]. Apoptosis can be divided in to intrinsic and extrinsic pathways. The intrinsic pathway can be activated by lysosomal leakage. Various compounds can be designed to specifically target lysosomes resulting in this death stimulus that can be used for therapeutic processes. The pathway downstream of lysosomal leakage is not well characterized. We were therefore interested in understanding the underlying molecular machinery leading up to cell death post leakage.

Three different compounds were chosen to stimulate lysosomal leakage: silica particles that are an occupational hazard causing lung fibrosis and the disease silicosis; staurosporine, which is a protein kinase inhibitor; and Leu-Leu-O-Me, which is a lysosmotropic agent. Alveolar macrophages were chosen as a cell line of choice due to their relevance to the development of silicosis. In all three instances, lysosomal leakage was measured using a FITC-dextran leakage assay. Lysosomal leakage was shown to activate the protein Bid. Bid activates cytoplasmic Bax resulting in its relocalization to mitochondria and the formation of pores in the outer mitochondrial membrane (MOMP). This leads to release of Smac and Cytochrome-c resulting in activation of caspase-9 and -3. Activation of the latter is a point-of-no return in apoptosis. MOMP results in disruption of mitochondrial function by causing mitochondrial membrane depolarization.

Spectrally distinct genetically encoded fluorescent probes for Bid, Bax, Smac and caspase were expressed in macrophages individually or in combination. Both Bid and caspase were Forster Resonance Energy Transfer (FRET) based probes. Mitochondrial membrane depolarization was determined using TMRE. Time-lapse imaging was performed either on Nikon A1R confocal or Nikon eclipse epi-fluorescent microscope fitted with multi XY stage and a temperature controlled enclosure from OKO labs. This allowed us a semi-high throughput-imaging scheme while ensuring cellular viability.

With all three compounds, lysosomal leakage occured within one hour and continued over time. Representative data for staurosporine treated cells is shown in Figure 1. FRET based Bid activation was observed post caspase activation rather than after lysosomal leakage. However, Bax activation preceded 
mitochondrial membrane depolarization (Figure 2) and upon MOMP a simultaneous release of Smac from mitochondria was observed (Figure 3). Our data therefore does not support the role of Bid in Bax activation but rather shows Bax activation to occur by yet an unknown trigger. Caspase activation and cell blebbing, a morphological characteristic of apoptosis was shown to occur simultaneously with mitochondrial depolarization in staurosporine treated cells or with mitochondrial hyperpolarization in silica treated cells. Time-lapse microscopy when used with appropriate fluorescent proteins can provide answers that may not be possible with population analysis techniques like flow-cytometry because it allows the sequential nature of processes to be examined on a cell-by-cell basis. The method described here has a potential to be applied to 96-well plate format further scaling up the throughput. The potential for multiplexing different probes and test compounds is endless. Cellular imaging along with appropriate data mining tools can provide both qualitative and quantitative output where biological activity of various targets can be compared and their interactions with other cellular targets can be visualized.

\section{Reference:}

1. Galluzzi, L. et al. Cell Death Differ 19, 107-120 (2012).

Figure 1: Staurosporine induced lysosomal leakage using FITC-dextran as demonstrated by an increase in green FD cellular fluorescence over time

Figure 2: Temporally distinct Bax redistribution and mitochondrial membrane depolarization in a cell population treated with staurosporine. Bax re-distribution from cytoplasm to mitochondria occur at 321 minutes for cell 1 where as at 352 minutes for cell 2 as indicated by arrows. It took 30 minutes post MOMP for mitochondrial membrane depolarization as observed in TMRE channel
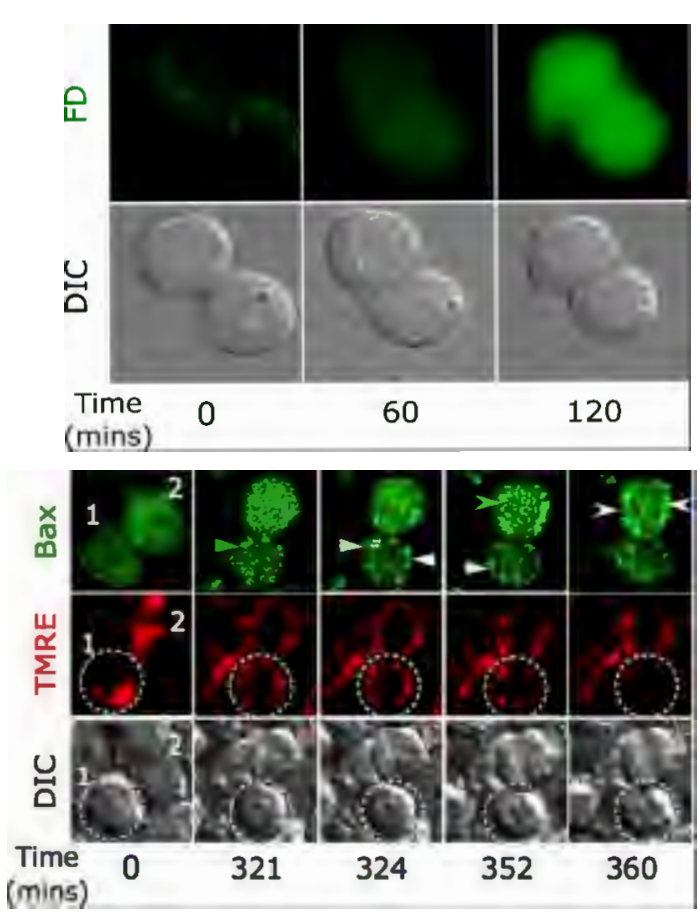

Figure 3: Bax redistribution to mitochondira and release of Smac from mitochondria occur simultaneously

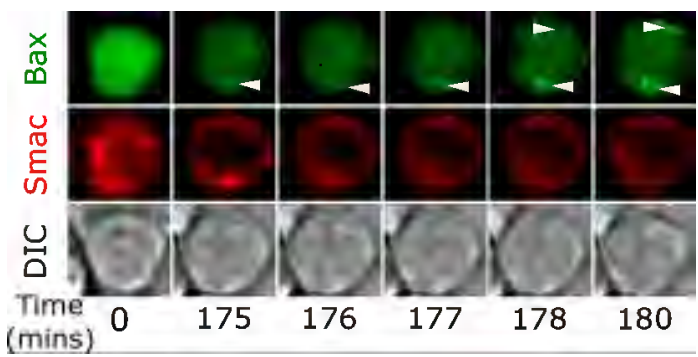

